\title{
Splenic Niche Cells from Young Heterochronic Parabionts Have Decreased Capability to Amplify T-cell Proliferation in Vitro
}

\author{
Dmytro W. Shytikov, ${ }^{1,}$, Maryna S. Shkumat ${ }^{1}$, Tetiana M. Yankova ${ }^{1}$, Alex G. Peregudov², \\ Igor V. Artyuhov", Iryna M. Pishel ${ }^{1}$ \\ ${ }^{1}$ D. F. Chebotarev State Institute of Gerontology National Academy of Medical Sciences of Ukraine, Kyiv, Ukraine \\ ${ }^{2}$ Institute of Biology of Aging, Moscow, Russia
}

\section{Email address:}

shytikov.dmitrij@gmail.com (D.W. Shytikov), marina_yuzik@mail.ru (M. S. Shkumat), yankovatetyna@ukr.net (T. M. Yankova), alex.peregudov@gmail.com (A. G. Peregudov), igor.artyuhov@gmail.com (I. V. Artyuhov), iryna.pishel@gmail.com (I. M. Pishel)

\section{To cite this article:}

Dmytro W. Shytikov, Maryna S. Shkumat, Tetiana M. Yankova, Alex G. Peregudov, Igor V. Artyuhov, Iryna M. Pishel. Splenic Niche Cells from Young Heterochronic Parabionts Have Decreased Capability to Amplify T-cell Proliferation in Vitro. American Journal of BioScience. Vol. 3, No. 2, 2015, pp. 46-54. doi: 10.11648/j.ajbio.20150302.14

\begin{abstract}
Immune system dysfunction during aging is well documented fact. Changes in the adaptive immune system are the most marked, especially in the T-cell compartment. In multiple preliminary studies it has been shown that parabiosis between 2 animals of different age can induce age-related changes in adaptive immune response and T-cell subpopulation composition of younger animal. In present study we evaluated the age-related changes in functions of rapidly renewing (macrophages) and slowly renewing cells $\left(\mathrm{CD} 11 \mathrm{c}^{+} \mathrm{DCs}\right)$ cells in spleens of young and old parabionts. We observed impaired capacity of splenic adherent cells from younger heterochronic parabionts to co-stimulate proliferation of autologous T-cells in vitro up to the level of old animals. Also we observed negative effect of this splenic cell population on intracellular signaling mechanisms that regulate PHA-activated proliferation of T-cells from young animals: statistically significant decrease of NFKB p65 expression and increased expression of IKB $\alpha$ during early activation events. This fact suggests that splenic macrophages may be involved in the induction of age-related changes of the immune system and they can be prospective target for further investigation.
\end{abstract}

Keywords: CD11c ${ }^{+}$Dendritic Cells, Macrophages, Heterochronic Parabiosis, Lymphoid Niche, Aging

\section{Introduction}

Aging leads to dysfunctions of various organs and systems in an organism. The earliest and the most pronounced agerelated changes are associated with the immune system. Impairment in functions of immune system with age results in decrease of the protective potential of an organism against both extrinsic and intrinsic pathogens [1].

Adaptive immune response most markedly declines with age and main reasons for that is atrophy of the thymus that begins immediately after puberty and massive changes in the T-cell compartment of lymphoid organs [2]. Aged T-cells often have a declined proliferative capacity and exert impaired effector's functions [3]. Aged naive T-cells also have defects in their proliferation and activation [4]. Defects in T-cell functions greatly affect protective capabilities of an organism so that it becomes more susceptible to different pathogens and cancer.

Numerous attempts to rejuvenate the immune system have shown that processes of age-related changes in T-cell functions are rather complex. Moreover, multiple studies done by Butenko and colleagues showed that a contact between the young immune system and the old one established via the common blood cell circulation induced an age-related decline in the young immune system [5]. Induction of age-related changes in some other organs such as aorta, skin [6], and brain was also demonstrated in a few studies on heterochronic parabiosis [7].

Earlier we investigated mechanisms of changes in the Tcell lymphoid compartment in young heterochronic partners 
during parabiosis [8]. We found that age-dependent changes in functions of the young immune system start with a disorder of the T-lymphocyte subpopulation in the spleen. These changes cannot be explained just by a transfer of the blood soluble factors, since lethal irradiation with X-rays of older animals in heterochronic parabiotic pair could partially preserve functional parameters of immune system of younger animals [9]. Therefore we assumed that these changes could be caused by disorders of the homeostatic T-cell differentiation and mediated either by the lymphoid niche of old partners, with which young cells interact during migration, or by age-related changes in young partners' niches.

It is well-known that peripheral T-cell functions are regulated by multiple extrinsic factors, in particular by the microenvironment of lymphoid organs, where T-cells usually form special zones such as a periarteriolar lymphoid sheath in the spleen. T-cell activities in these zones are regulated by cells of their niche. The latter include fibroblastic reticular cells, which organize T-cell zones, and antigen-presenting cells (APCs). APCs are important T-cell regulators and include a broad variety of different cell types such as dendritic cells, B-cells and macrophages. Dendritic cells (DCs) are professional APCs and regulatory cells and are able to either induce tolerance or stimulate profound immune response in an organism [10]. Macrophages can also display some DC-like features [11]. APCs are comprised of a very heterogeneous population of cells and they all can significantly affect T-cell functions. APCs can regulate T-cell functions through multiple mechanisms, particularly through the action of paracrine factors (cytokines) and through costimulatory or inhibitory surface molecules.

However little is known about lymphoid microenvironment changes during aging and its influence on T-cell functions. The main difficulty is that the niche is formed by various cell types with different functions, different lifetimes and varying migration degrees. Therefore the lymphoid niche in heterochronic parabionts is better to be presented by cell populations with different degrees of chimerism. It was communicated that in young isochronic parabiotic mice B-cells, T-cells and stationary monocytes/macrophages in blood underwent a complete exchange by day 9 after the initiation of parabiosis whereas the DC exchange reached plateaus of $\sim 24.4 \%$ only on day 30 after the surgery [12]. So the goal of this experiment was to evaluate the degree of age-related changes in functions of cell populations of rapidly renewing (macrophages) and slowly renewing cells (DCs) in the spleen from parabionts of different age.

\section{Materials and Methods}

\subsection{Mice and Parabiosis}

$\mathrm{CBA} / \mathrm{Ca}$ mice were bred and maintained at the Animal Research Facility of D.F. Chebotarev State Institute of Gerontology. For parabiosis, animals (females) were typically joined at 2-3 (young) and 22-23 (old) months of age. A parabiosis surgery was performed exactly as described previously [8] and in accordance with the guidelines of the Administrative Panel for Lab Animal Care of the Institute. Six weeks after the initiation of parabiosis, mice were euthanized and subjected to a tissue analysis.

\subsection{Experimental Groups}

The parabionts were divided into the following experimental groups: heterochronic parabionts that include young and old partners (designated on figures as Yo and Oy) and young and old isochronic parabionts (designated on figures as $\mathrm{Yy}$ and $\mathrm{Oo}$ ) as controls of corresponding heterochronic group. All parabiotic pairs, where animals had visible tumors of internal organs, had been excluded from the study.

\subsection{Isolation of Cellular Populations}

Spleens were removed from mice and disrupted by mechanical dissociation in $1 \mathrm{ml}$ of the complete RPMI-1640 medium with glutamine, penicillin $(100 \mathrm{U} / \mathrm{mL})$, streptomycin (100 $\mu \mathrm{g} / \mathrm{mL})$, HEPES $(25 \mu \mathrm{mol} / \mathrm{mL}$, Sigma-Aldrich), and $7,5 \%$ heat-inactivated FCS (Sangva, Ukraine). Total populations of splenocytes, splenic T-cells, macrophages, and dendritic cells were isolated by standard methods (See below) and analyzed.

T-cells were isolated from the total suspension of splenocytes using columns with nylon wool (Polyscience, Inc.) according to the manufacturer instruction. Flow cytometry analysis of enriched T-cell suspension (stained by anti-CD3-FITC monoclonal antibody (Pickcell Laboratory, Netherlands)) indicated $90-95 \%$ purity.

Dendritic cells were isolated from the spleen by a CD11cmagnetic separation kit EasySep (Stemcell Technology) according to the manufacturer instruction. The spleen was cut to 2 pieces. One half was used for the T-cell isolation. Another half was digested by collagenase D and the cell suspension was used for the magnetic bead cell sorting. Then the cells were counted and the suspension was added to the suspension of T-cells for a further analysis.

The total population of enriched splenic macrophages was harvested from the splenocytes by adhesion to plastic according to the routine method [8] with a few modifications. Splenocytes were obtained immediately after the disaggregating of the spleens in the complete RPMI-1640 medium. Splenocytes were counted and $15 \times 10^{6}$ cells in 500 $\mu 1$ of the complete RPMI-1640 medium were placed in 96well round-bottomed plates for 2 hours at $37^{\circ} \mathrm{C}$. Nonadherent cells were removed by several washes with the RPMI-1640 complete medium.

\subsection{Proliferation Assays}

Splenocytes or purified T-cells were cultured in the complete RPMI-1640 medium. In stimulation assays, the medium was additionally supplemented with phytohemagglutinin P (PHA-P; $10 \mu \mathrm{g} / \mathrm{mL}$, Sigma-Aldrich). 
Splenocytes were seeded $\left(2 \times 10^{6}\right.$ cells in $200 \mu 1$ of the culture medium) in 96-well round-bottomed plates (Sarstedt, Germany). Previously isolated T-cells were seeded $\left(1 \times 10^{6}\right.$ cells in $200 \mu$ of the culture medium) in similar plates for the proliferation assay. The plates were incubated in a humid environment with $5 \% \mathrm{CO}_{2}$ at $37{ }^{\circ} \mathrm{C}$ for 3 days. During coculture experiments previously isolated T-cells were additionally supplemented with either splenic dendritic cells or with splenic macrophages in the above-mentioned dilutions.

Cell proliferation was evaluated by the MTT colorimetric assay [13]. Proliferation rate was calculated as stimulation indexes (SI) by dividing OD values measured for stimulated cultures by those measured for unstimulated cultures (the mean of two replications):

$$
\mathrm{SI}=\mathrm{OD}_{\text {(stimulated cells) }} / \mathrm{OD}_{(\text {unstimulated cells })}
$$

\subsection{Co-Culture Experiments}

Co-culturing experiments were run to examine a capability of total splenic macrophages and dendritic cells to costimulate T-cell proliferation

Co-culture experiments included the following combinations:

1 Purified splenic T-cells from parabionts were cocultured with enriched population of autologous splenic dendritic cells.

2 Purified splenic T-cells from parabionts were cocultured with enriched population of autologous splenic macrophages.

Splenic dendritic cells and T-cells were prepared as described above. $1 \times 10^{6}$ of T cells for co-culture experiments were placed onto standard 96-well round-bottomed plates. Next, $0.01 \times 10^{6}$ of dendritic cells were added to the same well according to the appropriate co-culture standard procedure and the proliferation assay was done. Macrophages were prepared as described above in the same 96-well roundbottomed plates to be used for subsequent proliferation assays (Sarstedt, Germany). Then splenic T-cells were added to this well with the total population of splenic macrophages and the proliferation assay was done.

\subsection{Western Blotting}

T-cells analyzed for changes in their molecular mechanisms of activation were co-cultured with macrophages as reported above. $5 \times 10^{6}$ T-cells were cocultured with the total population of splenic adherent cells isolated as described above. Subsequently, T-cells were cultured in the complete RPMI-1640 medium in the presence or absence of phytohemagglutinin P (PHA-P; $10 \mu \mathrm{g} / \mathrm{mL}$, Sigma-Aldrich) in round-bottomed tubes. T-cells were stimulated for 2 or 18 hours according to the co-culture standard procedure and then used in the experiment.

Cell lysates were prepared as described in [14]. T-cells after stimulation were incubated in the lysis buffer (150 mmol NaCl, 20 mmol TRIS, $10 \%$ glycerol, $1 \%$ Triton $\mathrm{X}$ 100, 5 mmol EDTA, $50 \mathrm{mmol} \mathrm{NaF,} \mathrm{1mmol} \mathrm{PMSF)} \mathrm{for} 30$ min on ice. Then they were centrifuged at $12000 \mathrm{~g}$ during 12 min at $+4^{\circ} \mathrm{C}$ and the obtained cellular lysates were frozen at $-20^{\circ} \mathrm{C}$ for a further analysis. Before placing on gel, the protein concentration in the cellular lysates was measured with the colorimetric assay. The samples were then denaturized in the Laemli buffer with 5 min boiling and placed on gel. Proteins were transferred on PVDF membranes (Carl Roth $\mathrm{GmbH}$ ) according to the manufacturer instruction.

The membranes were probed with polyclonal rabbit antimouse antibodies (Santa Cruz) against: NFкB p65 RelA (sc372 ), caspase 3 (sc-7148), I $\kappa \mathrm{B} \alpha$ (sc-371). $\beta$-Actin (Abcam, ab-8227) was used as a reference protein. Activation of caspase 3 was determined by the detection of its mature p20 form. $\alpha$-Rabbit polyclonal antibodies conjugated with horseradish peroxidase (Sigma Aldrich) were used as secondary antibodies. The visualization was done with the use of a standard ECL system; chemiluminiscence was detected using X-ray films (AGFA). Same membranes were probed for the aforesaid antibodies in the consecutive order. Band detection and densitometric analysis were done with GelAnalyzer 2010a. Normalization of densitometric data of certain sample was done relatively to the $\beta$-Actin band and then relatively to the relative expression value of corresponding protein of the reference sample on the same membrane. As reference samples were used lysates of T-cells from young isochronic parabiont.

\subsection{Statistical Analysis}

Statistical analysis was done with the use of unpaired Student t-test and Mann-Whitney U-test. Values are presented as mean value $\pm \mathrm{SE}$. All operations were performed using Statistica 7.0.

\section{Results}

In the previous study we found that changes in the composition of T-cell subpopulations in the spleen of young heterochronic parabionts occurred as early as at 6 weeks of parabiosis, so it is possible that these changes may be mediated by disorders in the functions of cells that form the splenic T-cell niche. To investigate changes in functional state of some cell populations that form the microenvironment of the spleen $\left(\mathrm{CD} 11 \mathrm{c}^{+}\right.$cells and macrophages) during heterochronic parabiosis was the main aim of this investigation.

Age-related changes in functions of APCs are subject of great debates [15] and data about it is controversial. As APCs orchestrate activation of T-cells through the vast array of contact and soluble factors, investigation of particular signaling mechanism can lead to improper appraisals. To determine overall effect of heterochronic parabiosis on APCs we have chosen an in vitro model of PHA-mediated T-cell stimulation in presence of particular type of APCs - either total population of $\mathrm{CD} 11 \mathrm{c}^{+}$splenic dendritic cells or enriched by macrophages population of splenic adherent cells. During this test T-cells are stimulated via binding of PHA, T-cell 
superantigen, with T-cell receptor what results in polyclonal activation of T-cells. This way of T-cell in vitro stimulation is simple, reliable and physiological instrument of measuring Tcell antigen-induced proliferation because it mimics binding of presented antigen with T-cell receptor [16], not involving direct artificial activation of intracellular signal cascades. Thus it reflects more properly functional state of T-cells then T-cell stimulation with PMA or monoclonal anti-CD3 antibodies.

To investigate basal changes in the proliferative capacity of T-cells itself, without influence from tested populations of APCs, we have tested PHA-mediated proliferative response of complete splenocyte population or purified T-cells alone.

We stimulated splenocytes with PHA and observed significant differences between young and old isochronic parabionts (FIGURE 1A, $\left.\mathrm{P}_{(\mathrm{t})}<0,05\right)$. The splenocyte proliferation rate did not differ in young and old heterochronic parabionts as compared to either young or old isochronic controls. Changes in this parameter were not statistically significant and only tendentious in young heterochronic partners in this term $(1.5$ months of coexistence) of parabiosis.
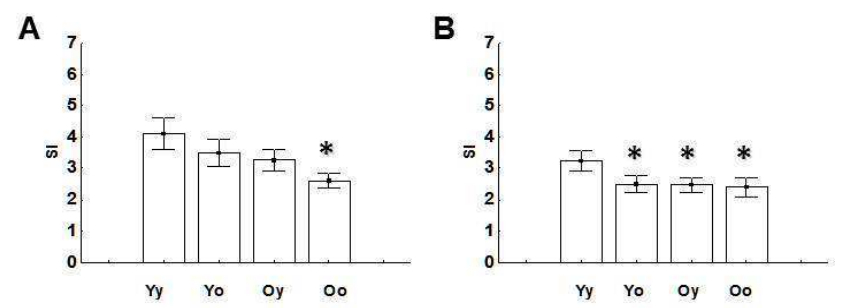

Figure 1. Impaired proliferative response to PHA of splenocytes (A) and purified splenic T-cells (B) of heterochronic parabionts comparing to young isochronic parabionts after 6 weeks of parabiosis.

Stimulation indexes (SI) were measured with MTT-test. Data is presented from results of at least six experiments.

* $-\mathrm{P}_{(\mathrm{t})}<0,05$ comparing to young isochronic parabionts.

We used nylon wool for isolation and enrichment of splenic T-cells. We observed a great decrease in the proliferative capacity of enriched population of splenic $\mathrm{T}$ cells from young heterochronic parabionts and no signs of its correction in old heterochronic partners (FIGURE 1B, $\mathrm{P}_{(\mathrm{t})}<$ $0,05)$. So, we observed presence of impairment in proliferation in vitro of T-cells from young heterochronic parabionts after 1.5 months of the co-existence.

It is known that the function of T-cells depends much on their microenvironment in lymphoid organs, particularly on function of antigen-presenting cells. We tested effects of total population of splenic dendritic cells and macrophage-rich population of splenic adherent cells on proliferative response of purified splenic T-cells since they could be considered as possible key regulators of T-cell functions. As interaction of T-cells usually involve great number of soluble and surface co-stimulatory molecules we decided not test expression of particular molecule as such approach might result in underestimation of one factors and overestimation of another ones. Instead we decided to test the summary effect of co- cultivation of enriched populations of splenic dendritic cells or enriched with macrophages population of total splenic adherent cells on PHA-induced T-cell activation in vitro.

We identified a significant decrease in the proliferation rate of T-cells from old isochronic parabionts co-cultured with autologous dendritic cells as compared to the young isochronic ones (FIGURE 2A, $\left.\mathrm{P}_{(\mathrm{t})}<0,05\right)$. Meanwhile no decrease was observed in the proliferation rate of T-cells cocultured with total splenic dendritic cells from young heterochronic parabionts to the level of young isochronic parabionts. We also analyzed influence of the total population of splenic adherent cells from animals of different experimental groups on the $\mathrm{T}$ cell proliferation in vitro (FIGURE 2B). The proliferative capacity of $\mathrm{T}$ cells from young heterochronic partners which were co-cultured with their own splenic adherent cells was found to drop to the level of old animals as comparing to young isochronic partners $\left(\mathrm{P}_{(\mathrm{t})}<0,05\right)$. Thus we suggest that the T-cell proliferative capacity can be strongly impacted by nicheforming cells and their age-related changes can cause T-cell dysfunction. And macrophages could act as T-cell dysfunction inducers in our experimental model of heterochronic parabiosis.

Analysis of influence of splenic macrophages on the molecular mechanisms of T-cells' activation could give additional information about changes in functions of splenic macrophages during heterochronic parabiosis.
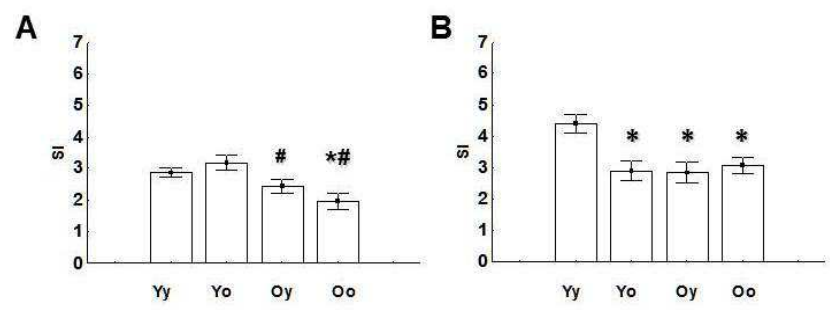

Figure 2. Changes in the capability of dendritic cells and macrophage-rich population of adherent cells of experimental animals to costimulate the proliferation of splenic T-cells from the same animals in vitro.

Total population dendritic cells and macrophages were isolated from spleens of animals of different experimental groups according to the standard procedure. Total population of splenic T-cells from animals of the same experimental groups were isolated with the help of nylon wool and stimulated with PHA in the presence of previously isolated splenic dendritic cells (A) or macrophage-rich population of adherent cells (B). Stimulation indexes (SI) were measured with MTT-test. Data is presented from results of at least six experiments.

* $-\mathrm{P}_{(\mathrm{t})}<0,05$ comparing to young isochronic parabionts

$\#-\mathrm{P}_{(\mathrm{t})}<0,05$ comparing to young heterochronic parabionts

We decided to analyze key mechanisms of T-cell activation because it is often characterized by changes in multiple regulatory mechanisms and $\mathrm{NF \kappa B}$ p65 plays essential role during activation of T-cells [17]. Its decreased or aberrant expression may cause impaired functions of T-cells. According to this we decided to test expression of NFкB p65 during activation of T-cells. We analyzed 2 time points of Tcell stimulation -2 and 18 hours what can be addressed to relatively short and long terms of stimulation. 
As the most striking differences in T-cell proliferation were observed in experiment with its co-culturing with the splenic macrophages, we analyzed their influence on T-cell molecular signaling during activation. The analysis of the expression level of key molecules engaged in T-cell

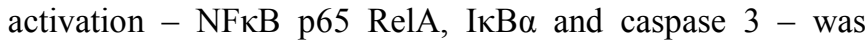
performed by western blotting.

After 2 hours of cocultivation of enriched T-cell population with macrophages from spleens of autologous donors (coculture Scheme 2) we observed decreased level of RelA and caspase 3 p 20 protein in old isochronic parabionts versus the young isochronic ones (FIGURE 3A and 3C, respectively, $\left.\mathrm{P}_{(\mathrm{U})}<0,05\right)$. In young heterochronic parabionts, T-cell cocultivation with macrophages led to another effect - the
RelA level also reduced whereas caspase 3, p20 protein remained unchanged in this group (FIGURE $3 \mathrm{~A}$ and $3 \mathrm{C}$, respectively, $\left.\mathrm{P}_{(\mathrm{U})}<0,05\right)$. Neither significant changes in the I $\kappa \mathrm{B} \alpha$ level (FIGURE 3B) nor any sign of expression of the full (not active) form of caspase 3 in T-cells were detected (data not presented). However noteworthy is an interesting fact: T-cells both from young isochronic and from young heterochronic parabionts expressed a markedly higher level of the activated form of caspase 3 p20 protein (FIGURE 3C, $\left.\mathrm{P}_{(\mathrm{U})}<0,05\right)$, which in general may be considered as a negative process, that oppose to proliferation, though T-cells from young isochronic parabionts proliferated with a much higher rate than T-cells from other experimental groups.
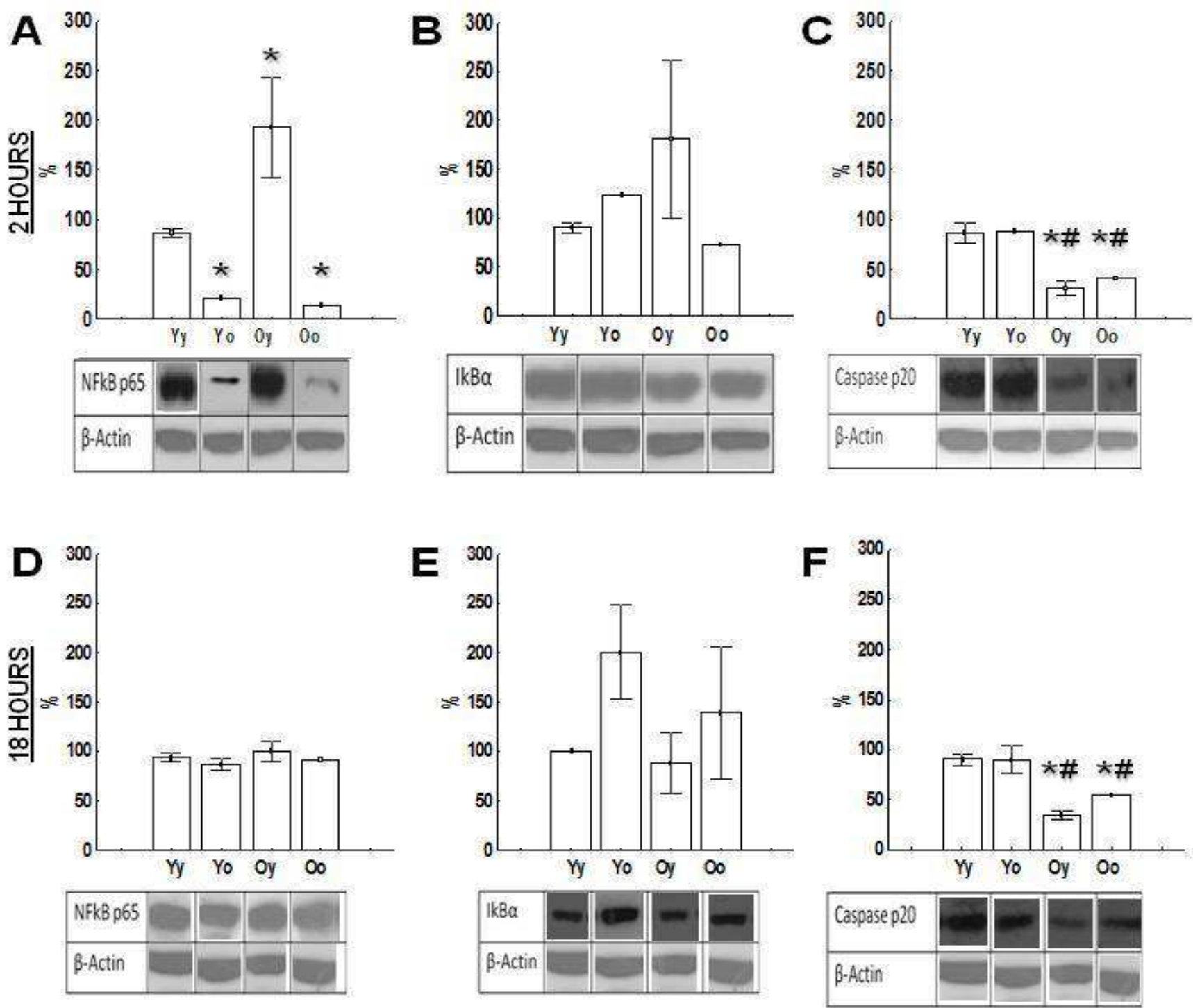

Figure 3. Changes in the molecular signalling of splenic T-cells from parabiotic mice under influence of total population of splenic macrophage-rich population of adherent cells from animals of the same experimental groups.

Total splenic T-cells from parabionts of different experimental groups isolated and $10 * 10^{6}$ were cocultured with previously isolated total splenic macrophages from $10 * 10^{7}$ splenocytes of animals of the same experimental groups. $20 \mu \mathrm{g} / \mathrm{ml} \mathrm{PHA}$ were added to cell suspensions and they had been stimulated for 2 (A, B, C) or $18(\mathrm{D}, \mathrm{E}, \mathrm{F})$ hours. Then cells were lysed and total cellular extract had been analyzed with Western Blotting for the main components of T-cell activation signalling: RelA (A, D), IkB $\alpha(B, E)$, caspase p20 (C, F). Data is presented from results of at least three experiments.

* - $\mathrm{P}_{(\mathrm{U})}<0,05$ comparing to young isochronic parabionts

$\#-\mathrm{P}_{(\mathrm{U})}<0,05$ comparing to young heterochronic parabionts 
After 18 hours of cocultivation we observed equalization of the RelA protein level between T-cells from all experimental groups (FIGURE 3D). No considerable changes in the expression level of the $\mathrm{I} \kappa \mathrm{B} \alpha$ protein in activated T-cells from different experimental groups were marked in this stage of activation (FIGURE 3E). However the level of the activated form of caspase 3 (p20) was still considerably higher in T-cells from young isochronic and young heterochronic parabionts in comparison with T-cells from either old isochronic or heterochronic parabiotic animals (FIGURE 3F, $\left.\mathrm{P}_{(\mathrm{U})}<0,05\right)$.

The experiment focused merely on the key factors of T-cell activation during APCs co-stimulation, and we found out considerable changes in the expression level of several key proteins for T-cell mitogen-induced proliferation. We notice that splenic macrophages can impact T-cell proliferation and mitogen-activated T-cell signaling.

\section{Discussion}

In our previous study we have shown that common circulation arranged between animals of different ages enables the penetration of young stem cells, naive T-cells, hormones, and growth factors into an old organism and doesn't bring about any rejuvenation of the old mice' immune system in the heterochronic parabiosis model. On the contrary, the differentiation of young T-cells featured some changes: a lower number of $\mathrm{CD}^{+}$cells, a lesser CD4/8 ratio in the spleen and a higher number of memory-like $\mathrm{CD}^{+} 44^{+}$cells. These changes in the composition of the Tcell subpopulation ultimately led to a decrease in mitogeninduced T-cell proliferation and immune response in young heterochronic partners $[5,8,9]$. We presume that all the changes are likely to be influenced by changes in the functions of splenic niche of young heterochronic partners [8]. To verify this hypothesis in the present research the costimulatory activity of some cell populations that form the microenvironment of the spleen $(\mathrm{CD} 11 \mathrm{~s}+$ cells and macrophages) was tested.

It has been well-known that functioning of T-cells greatly depends on their niche, and APCs being its most essential component. They include B cells, macrophages and dendritic cells. The last are considered as the most potent antigenpresenting cell type [16]. A role of APCs in T-cell functioning is hardly to be overestimated - they regulate activation and differentiation of T-cells, shape the TCR repertoire and assist in the cessation of unimportant immune response. Interactions of APCs with T-cells include TCR-MHC coupling, a contact through an array of surface molecules (adhesion, co-stimulatory and co-inhibitory molecules) and expression of multiple soluble molecules (interleukins and cytokines).

Primary interactions of APCs and T-cells include TCR and MHC pairing [19]. It can result in either activation of a T-cell (in the case of specific recognition) or in sub-optimal stimulation of T-cells, which is important for their survival, especially for $\mathrm{CD}^{+}$cells [20]. A renowned fact is that a sole primary TCR-MHC interaction is not enough for normal Tcell activation and induction of effective T-cell proliferative response.

The most frequently studied type of APCs is dendritic cells. However, data on dendritic cell age-related changes remains very contradictory [21]. Changes in the DC capacity to stimulate T-cells' proliferation with age have been so far unclear. Also, an assumption on a reduction in proliferative response of T cells co-cultured with aged DCs was made [22]. However recent studies do not suggest any reduction of $\mathrm{T}$ cell co-stimulation with aged APCs and a possible role of defects properly in aged T-cells, what is summarized in the review by Weng et al [23]. Summing up, it should be noted that age-related changes in functions of DCs, especially of splenic resident DCs, are currently a subject of debates.

The key objective of this research was to determine changes in one of the main functions of splenic APCs capability to influence on T-cell proliferation - after heterochronic parabiosis. Basing on the above data, we tested changes in myeloid elements of the lymphoid niche, particularly in the total population of splenic dendritic cells and in the total population of splenic adherent cells.

In our experiment we have tested $\mathrm{T}$-cell proliferative response to PHA in vitro in presence of dendritic cells or macrophages. We purified total fraction of splenic T-cells from parabionts of different experimental groups and stimulated them with PHA. The proliferation rate of the Tcells from young isochronic parabionts proliferated at significantly higher rate than T-cells from animals in other experimental groups (FIGURE 1B). This data suggests negative changes in T-cells of young heterochronic parabionts. Data of other authors evidences to almost $50 \%$ exchange of T-cells between parabiotic partners [12]. So, changes in the proliferative capacity could be explained either by changes in T-cells or by changes in their nicheforming cells.

It is quite a challenge to investigate functions of cells that form the lymphoid niche because of their extremely low quantity in lymphoid organs in spite of their important role in immune response. The changes in the number of these cells or in parameters such as expression of particular proteins can lead to a big error in evaluations. So, we originated from their total capacity to influence on proliferations of T-cells evaluating differences in their influence on the proliferation rate of T-cells. For that, we came up with an in vitro system with splenic T-cells from animals of different experimental groups and different components of the splenic lymphoid niche (e.g. macrophage-enriched population of splenocytes (adherent cells) and the total population of splenic CD11 ${ }^{+}$ cells) of same experimental groups to test the functional state of niche-forming cells.

In our experiment, T-cells from young isochronic parabionts co-cultured with autologous DCs had significantly higher proliferation rate than T-cells from old isochronic parabionts (FIGURE 2A, $\left.\mathrm{P}_{(\mathrm{t})}<0,05\right)$. No decrease was 
detected in the proliferation rate of T-cells from the young heterochronic parabionts co-cultured with own total population of $\mathrm{CD}_{11 \mathrm{c}^{+}}$splenic cells (FIGURE 2A) as compared to young isochronic parabionts. Also, we observed a much higher $\left(\mathrm{P}_{(\mathrm{t})}<0,05\right)$ proliferation rate of T-cells from young heterochronic parabionts compared with old isochronic and old heterochronic. However no significant differences were observed in the T-cell proliferation rate between T-cells from young isochronic and heterochronic parabionts co-cultured with autologous DCs. This fact may indicate that the functional activity of dendritic cells of the spleen varies slightly in young partners in heterochronic parabiosis.

Macrophages are another cell type that is likely to be of importance in T-cell niche functioning. They play a prominent role in numerous processes. In normal conditions, macrophages are responsible for tissue remodeling, maintaining tissue architecture and clearance from apoptotic cells and, furthermore, macrophages play a crucial role in fighting pathogens at inflammation sites [24]. Many macrophage types are essential for various immunological reactions and can provide other cell types with different cytokines [25]. There is evidence that macrophages also play an important role in the antigen presentation [26].

Investigations of age-related changes in macrophages are greatly complicated by the presence of multiple differentiation states and by distinctions between macrophages from different locations. The main reported age-related changes in macrophage functioning include decreased response to pathogens and increased basal expression of pro-inflammatory cytokines [27], which contributes greatly to the general inflammatory phenotype that is observed during aging; decreased killing of intracellular pathogens and decreased capture of antigens [28]; and changes in expression of effector's molecules [29]. Birjandi and colleagues [30] suggests that the splenic structural arrangement undergoes disorganization and negative functional changes occur in several macrophage populations.

Resident lymphoid dendritic cells, mostly, are unable to extensively migrate between partners, as shown by Swirski and colleagues [12]. Alternatively, macrophages can migrate into different sites through the bloodstream due to their direct progenitors - monocytes [31]. So, assumingly, DCs in general represent mostly nonmigrating fraction of the lymphoid niche cells, and macrophages are more mobile lymphoid niche element. A classical method for macrophage isolation is their adherence to the substrate. In our previous work we isolated spleen macrophages using their ability to adhere to plastic, and indicated that more than $50 \%$ of the adherent cells of the spleen are active phagocytic macrophages. And we noted a decrease in phagocytic activity of macrophages from all experimental groups relative to young isochronic parabionts [8]. Here a strong effect of macrophages on T-cell co-stimulation is observed in our in vitro model. As monocytes have a higher potential for migration between partners than DCs [12], we may assume that this effect may be caused by adherent cells from mixed aged and young donors. But this hypothesis needs to be tested in the future.

No decrease was detected in the proliferation rate of $\mathrm{T}$ cells co-cultured with total splenic dendritic cells from young heterochronic parabionts to the level of young isochronic parabionts, so it is possible to conclude that age-related changes in the co-stimulatory properties of the total population of dendritic cells did not take place in age-related immune cell disorders in this model. This can be explained by the fact that during parabiosis exchange with dendritic cells between partners is not active [12].

In our experiment we found a significant reduction in proliferation of T-cells from all experimental groups (compared with young isochronic animals) after their coculturing with autologous splenic adherent cells (FIGURE 2B). This fact may suggests negative changes in the splenic adherent cell population of young heterochronic partners, which can act as one of factors in the T-cell proliferative response impairment in later terms of parabiosis in particular, and on immune response in the main.

There is a wide array of co-stimulatory molecules for Tcells and each act in its particular way. Yet, regarding T-cell activation-induced proliferation, one of the main signaling events is to stimulate activation of the $\mathrm{NF \kappa B}$ family of transcription factors. It is well-known that proteins of the NFkB-transcription factors` family usually play a central role in T-cell activation and proliferation. Their correct functioning is important for T-cell activation-induced proliferation [17]. In a steady state, $\mathrm{NF \kappa B}$ factors persist in a cytoplasm as monomers bound to special inhibitory proteins. $\mathrm{NF} \kappa \mathrm{B}$ activation is opposed by several mechanisms and the key one is direct binding of monomeric NFKB proteins by inhibitory proteins, namely by an $\mathrm{I} \kappa \mathrm{B}$ protein. During activation of cellular proliferation $\mathrm{NF \kappa B}$ proteins form homo- and heterodimers, which in turn can activate transcription of genes needed for proliferation and inhibitory proteins of the I $\kappa \mathrm{B}$ family are subjected to the proteasomal degradation [32]. Great influence on it may be exerted by adequate co-stimulation derived from cells composing the Tcell niche. Cellular death can oppose proliferation, especially in the case of inadequate T-cell co-stimulation. However there are several mechanisms to induce T-cell death through co-stimulation. They play either a physiological or pathological role in functioning of the immune system. The exclusive role in this process belongs to apoptosis and its progression greatly depends on activation of caspase 3 , a member of the family of specific proteases that usually execute apoptosis, i.e. programmed cell death.

Phosphorylation and nuclear translocation of NFKBtranscription factors are critical events for T-cell activation and proliferation. But expression level of these regulatory proteins is also important factor for proper activation of Tcells. It has been reported that anergic T-cells often express lower amount of key signal or effector molecules. In a clinical study on patients with sarcoidosis blood $\mathrm{CD}^{+}$cells had impaired proliferation and reduced content of RelA and 
$\mathrm{p} 50$, which are part to NFאB transcription factors, lower expression of $\mathrm{p} 56^{\mathrm{Lck}}$-kinase, $\mathrm{CD} 3 \xi$ and a few other proteins, critical for T-cell activation. This can be partly caused by the functional exhaustion of T-cells and induction of the anergylike cell phenotype [33]. Similar results were obtained by Sunstedt and colleagues [34]. Anergic T-cells expressed a reduced amount of p65, AP1 and the c-fos transcription factor and expressed more p50. An analysis of expressed $\mathrm{NF} \kappa \mathrm{B}$ dimers revealed that $\mathrm{p} 50$ homodimers characterized as inactive were expressed in anergic T-cells at higher extent higher than p50-p65 heterodimers. Thus it has been established that in anergic T-cells activatory signaling is impaired [33] and decrease in expression of key regulatory proteins plays important role.

We have shown similar result in our model of influence of splenic macrophages on the proliferation rate of T-cells. Macrophages from young heterochronic parabionts and macrophages from old isochronic parabionts both provided weaker co-stimulation of T-cell proliferative response in vitro. In both cases we showed decreased expression of RelA in analyzed T-cells. It could be a possible mechanism of a decline in the functional properties of $\mathrm{T}$ cells in young heterochronic parabionts, what results in their reduced immune function in parabiosis. However we didn't observe significant differences in expression of $\mathrm{I} \kappa \mathrm{B} \alpha$ between animals of different experimental groups. Also, some differences, e.g. in expression of the activated form of caspase 3, were marked. Old animals had reduced expression of this protein in activated T-cells.

There is evidence that activation of caspase 3 probably plays a dual role in T-cell physiology. Caspase 3 is an executor caspase, which is activated during apoptosis and mediates cell death. On the other hand, activation of caspase 3 is necessary for T-cell proliferative response and without it $\mathrm{T}$ cell proliferation and activation is lower [35]. A mechanism of its action has been proposed - to some extent, it could be dependent on the inhibition of $\mathrm{I} \kappa \mathrm{B}$ proteins by fragments of partially digested RelA [36]. It is reported that T-cells from older animals displayed reduced sensitivity to apoptosis and a reduced turnover, what could be also some evidence of lower caspase activity [4]. However no truncated RelA form was fixed in our experiment whereas we did observe reduced expression of the activated form of caspase 3 in T-cells of old animals stimulated in presence of autologous macrophages. Reduced expression of the activated form of caspase 3 can be considered as a negative effect and a higher activation rate probably does not affect T-cell proliferation, as seen from McComb`s study [37]. However we did not observe any expression of the caspase 3 inactive forms in T-cells from young isochronic parabionts.

Summarizing, we have observed different influence of heterochronic parabiosis on capability of splenic nicheforming cells from young animals to stimulate proliferation of T-cells in vitro. It has been shown that ability of splenic CD $11 \mathrm{c}^{+}$dendritic cells to influence on the proliferation of T-cells varies slightly between young heterochronic and isochronic parabionts. But these changes are more evident in the fast-renewing population of splenic macrophages. We demonstrated decreased proliferative response of T-cells from young heterochronic parabionts when they were stimulated in vitro in presence of macrophage-rich population of autologous splenocytes. Also during stimulation in vitro in presence of autologous macrophages T-cells from young heterochronic parabionts had lower expression of NFKB p65 on early stages of stimulation (2 hours) and higher expression of $\mathrm{I} \kappa \mathrm{B} \alpha$ on later stages (18 hours) of stimulation comparing to T-cells from young isochronic parabionts. Observed data indicates induction of negative changes in functions of macrophage-rich population of splenocytes under the influence of heterochronic parabiosis.

\section{References}

[1] A. Larbi, G. Pawelec, S.C. Wong, D. Goldeck, J.J. Tai, T. Fulop. Aging of the immune system as a prognostic factor for human longevity. Physiology 2008; 23: 64-74.

[2] A. M. Holland, V. R. M. van den Brink. Rejuvenation of the aging $\mathrm{T}$ cell compartment. Curr. Opin. Immunol. 2009; 21: 454-9.

[3] S.L. Swain, J. Nikolich-Zugich. Key research opportunities in immune system aging. J. Gerontol. Biol. Sci. Med. Sci. 2009; 64: $183-6$

[4] H. Tsukamoto, K. Clise-Dwyer, G. E. Huston, D. K. Duso, A. L. Buck, L. L. Johnson et al. Age-associated increase in lifespan of nave CD4 T cells contributes to T-cell homeostasis but facilitates development of functional defects. Proc. Natl. Acad. Sci. USA. 2009; 106: 18333-8.

[5] G. M. Butenko, I. B. Gubrii. Inhibition of the immune responses of young adult CBA mice due to parabiosis with their old partners. Exp. Geront. 1980; 15: 605-10.

[6] Z. Deyl, G. M. Butenko, J. Hausmann, M. Horakova, K. Macek. Increased glycation and pigmentation of collagen in aged and young parabiotic rats and mice. Mech. Aging. Develop. 1990; 55: 39-49.

[7] S. A. Villeda, J. Luo, K. I. Mosher, B. Zou, M. Britschgi, G. Bieri et al. The ageing systemic milieu negatively regulates neurogenesis and cognitive function. Nature. 2011; 477: 90-4.

[8] I. Pishel, D. Shytikov, T. Orlova, A. Peregudov, I. Artyuhov, G Butenko. Accelerated aging versus rejuvenation of the immune system in heterochronic parabiosis. Rejuvenation. Res. 2012; 15: 239-48.

[9] G. M. Butenko, I. B. Gubriĭ. Mechanism of immune response inhibition during parabiosis of animals of different ages. Biull. Eksp. Biol. Med. 1981; 92: 318-9.

[10] N. Cools, P. Ponsaerts, V. F. Van Tendeloo, Z. N. Berneman. Balancing between immunity and tolerance: an interplay between dendritic cells, regulatory $\mathrm{T}$ cells, and effector T cells. J. Leukoc. Biol 2007; 82: 1365-74.

[11] D. A. Hume. Macrophages as APC and the Dendritic Cell Myth. J. Immunol. 2008; 181: 5829-35. 
[12] F. K. Swirski, M. Nahrendorf, M. Etzrodt, M. Wildgruber, V. Cortez-Retamozo, P. Panizzi et al. Identification of splenic reservoir monocytes and their deployment to inflammatory sites. Science. 2009; 325: 612-16.

[13] T. Mosman. Rapid colorimetric assay for cellular growth and survival: application to proliferation and citotoxity assay $\mathrm{J}$. Immunol. Metods. 1983; 65: 55-63.

[14] K. W. Kaufmann, C.I. Behe, V. M. Golubovskaya, L. L. Byrd, C. D. Albright, K. M. Borchet. Aberrant cell cycle checkpoint function in transformed hepatocytes and WB-F344 hepatic epithelial stem-like cells. Carcinogenesis 2011; 22: 1257-69.

[15] A. Agrawal, S. Agrawal, J. Tay, S. Gupta. Biology of Dendritic Cells in Aging. J. Clin. Immunol.2008; 28: 14-20.

[16] A. M. Kruisbeek, E. Shevach, A. M. Thornton. Proliferative Assays for T Cell Function. Current Protocols in Immunology. 2004; 60: III:3.12:3.12.1-3.12.20.

[17] J. E. Smith-Garvin, G. A. Koretzky, M. S. Jordan. T cell activation. Annu. Rev. Immunol. 2009; 27: 591-619.

[18] F. Geissmann, M. G. Manz, S. Jung, M. H. Sieweke, M. Merad, K. Ley. Development of monocytes, macrophages, and dendritic cells. Science. 2010; 327: 656-61.

[19] D. Fooksman, S. Vardhana, G. Vasiliver-Shamis, J. Liese, D. A Blair, J. Waite. Functional Anatomy of T Cell Activation and Synapse Formation. Annu. Rev. Immunol. 2010; 28: 79-105.

[20] O. Boyman, S. Létourneau, C. Krieg, J. Sprent. Homeostatic proliferation and survival of naïve and memory T cells. Eur. J. Immunol. 2009; 39: 2088-94.

[21] A. Agrawal, S. Gupta. Impact of Aging on Dendritic Cell Functions in humans. Ageing. Res. Rev. 2011; 10: 336-45.

[22] S. Komatsubara, B. Cinader, S. Muramatsu. Polymorphism of age-related changes in stimulatory capacity of murine dendritic cells. Mech. Ageing Dev. 1986; 37: 163-73.

[23] N. P. Weng, A. N. Akbar, J. Goronzy. CD28- T cells: their role in the age-associated decline of immune function. Trends Immunol. 2009; 30: 306-12.

[24] J. A. Stefater 3rd, S. Ren, R. A. Lang, J. S. Duffeld. Metchnikoff's policemen: macrophages in development, homeostasis and regeneration. Trends Mol. Med. 2011; 17: 743-52.

[25] D. M. Mosser, J. P. Edwards. Exploring the full spectrum of macrophage activation. Nat. Rev. Immunol. 2008; 8: 958-69.
[26] R. N. Barker, L. P. Erwig, K. S. Hill, A. Devine, W. P. Pearce, A. J. Rees. Antigen presentation by macrophages is enhanced by the uptake of necrotic, but not apoptotic, cells. Clin. Exp. Immunol. 2002; 127: 220-25.

[27] R. D. Stout, J. Suttles. Immunosenescence and macrophage functional plasticity: dysregulation of macrophage function by age-associated microenvironmental changes. Immunol. Rev. 2005; 205: 60-71.

[28] Y. Sun, H. Li, M. F. Yang, W. Shu, M. J. Sun, Y. Xu. Effects of Aging on Endotoxin Tolerance Induced by Lipopolysaccharides Derived from Porphyromonas gingivalis and Escherichia coli. PLoS ONE 2012; 7:e39224.

[29] C. A. Cecílio, E. H. Costa, P. U. Simioni, D. L. Gabriel, W. M. Tamashiro. Aging alters the production of iNOS, arginase and cytokines in murine macrophages. Braz. J. Med. Biol. Res. 2011; 44: 671-81.

[30] S. Z. Birjandi, J. A. Ippolito, A. K. Ramadorai, P. L. White. Alterations in marginal zone macrophages and marginal zone B cells in old mice. J. Immunol. 2011; 186: 3441-51.

[31] C. Shi, E. G. Pamer. Monocyte recruitment during infection and inflammation. Nat. Rev. Immunol. 2011; 11: 762-74.

[32] E. O'Dea, A. Hoffmann. The regulatory logic of the NFkappaB signaling system. Cold Spring Harb. Perspect. Biol. 2010; 2: a000216.

[33] N. S. Lee, L. Barber, A. Kanchwala, C. J. Childs, Y. P. Kataria, M. A. Judson et al. Low levels of NF- $\mathrm{BB} / \mathrm{p} 65$ mark anergic CD4+ T cells and correlate with disease severity in sarcoidosis. Clin. Vaccine. Immunol. 2011; 18: 223-34.

[34] A. Sundstedt, M. Sigvardsson, T. Leanderson, G. Hedlund, T. Kalland, M. Dohlsten. In vivo anergized CD4+ T cells express perturbed AP-1 and NF-kappa B transcription factors. Proc. Natl. Acad. Sci. USA. 1996; 93: 979-84.

[35] J. K. Norman, T. Kataoka, J. Tschopp, R. C. Budd. Caspase Activation Is Required for T Cell Proliferation. J. Exp. Med. 1999; 190: 1891-96.

[36] M. Coiras, M. R. López-Huertas, E. Mateos, J. Alcami. Caspase-3-mediated cleavage of p65/RelA results in a carboxy-terminal fragment that inhibits $\mathrm{I} \kappa \mathrm{B} \alpha$ and enhances HIV-1 replication in human $\mathrm{T}$ lymphocytes. Retrovirology 2008; 5: 109.

[37] S. McComb, R. Mulligan, S. Sad. Caspase-3 Is Transiently Activated without Cell Death during Early Antigen Driven Expansion of CD8+ T Cells In Vivo. PLoS One. 2010; 5: e15328. 